\title{
Correction to: Experimental and numerical study on transient elongational viscosity for PP/LDPE blends
}

\author{
Yasuhiko Otsuki • Yoko Fujii · Hiroko Sasaki • Panitha Phulkerd • Masayuki Yamaguchi
}

Published online: 9 January 2020

(c) The Society of Polymer Science, Japan 2020

\section{Correction to: Polymer Journal}

https://www.nature.com/articles/s41428-019-0286-0

In the original published version of Fig. 4, the contributions of the continuous PP phase and the dispersed LDPE phase to the numerical results of transient elongational viscosity were incorrect. The corrected figures are shown below. The original article has been corrected. 

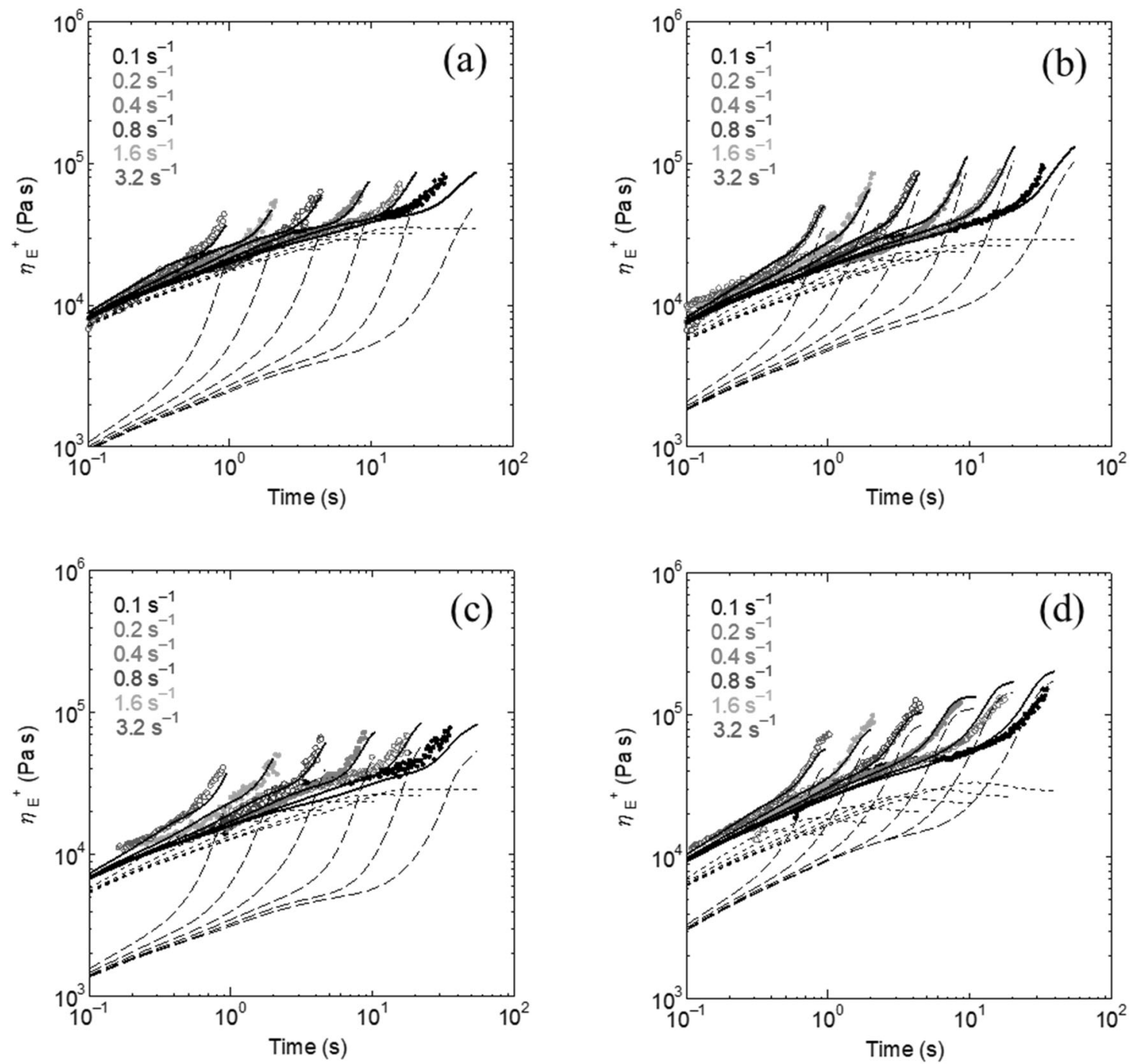

Fig. 4 Transient elongational viscosity $\eta_{\mathrm{E}}^{+}$with time at various Hencky strain rates at $190{ }^{\circ} \mathrm{C}$ for (a) PP/LDPE-M (85/ 15), (b) PP/LDPE-M (70/30), (c) PP/LDPE-L (70/30), and (d) PP/ LDPE-H (70/30). The solid lines represent the numerical results. The contributions of the stress generated in PP (dotted lines) and LDPE (dashed lines) are also indicated 